published elsewhere. We are grateful to Drs. $\mathrm{H}$. Steiner and E. T. Borrows for discussions, to Dr. A. Elliott for some of the infra-red absorption measurements, and Dr. Rubin and Mr. Street for experimental assistance.

${ }^{1}$ Staudinger and Brusson, Annalen, 447, 103, 110 (1926), and Carmondy, Sheehan and Kelly, Indust.' Eng. Chem., 30, 245 (1938) prepared polyeyclopentadienes and suggested repeating units as in (Ic) or (I $(d)$; it is possible that some of the polycyclopentadienes described in the recent patent literature are also built up according to this latter structural principle; cf., for example, Soddy, U.S.A.

${ }^{2}$ Cr. Weiss, J. Chem. Soc., 245 (1942).

- Hausser, Kuhn and Smakula, Z, phys. Chem., (B), 29, 384 (1935) Karrer and Jucker, "Carotinoide"" (Basle, 1948)." Blout and Fields, J. Amer. Chem. Soc., 70, 189 (1948).

- Rubin, Steiner and Wassermann,.J. Chem. Soc., 3046 (1949).

${ }^{5}$ Alder and Stein, Annalen, 504, 216 (1933).

'Nernst and Hohmann, $Z$. phys. Chem., 11, 352 (1893). Clark, Ph.D. thesis, University of Missouri (1937). Wassermann (unpublished experiments).

\section{PAPER CHROMATOGRAPHY OF MUSCLE TISSUE CONSTITUENTS Paper Chromatography of Adenosine Phosphates}

CARTER ${ }^{1}$ has devised a method for paper 4 chromatography of adenosine phosphates. He uses as solvent a 5 per cent dibasic sodium phosphate solution and iso-amyl alcohol. For developing the spots after drying, he sprays the paper with fluorescein and localizes the spots with an ultra-violet lamp ('Mineralight').

We have modified the partition coefficient between the two phases by using a solution of 0.5 per cent lauryl amine in $n$-amyl alcohol instead of iso-amyl alcohol. The resolution of the spots has much improved by this method. We believe that this method can be adapted to other substances; thus, for substances of basic character laurylic acid can be used to improve the resolution.

We have compared our method with the original method of Carter on adenosine phosphates. With Carter's method, using an adenylic acid preparation (Merck), two spots were obtained with $R_{F} 0.73$ and $R_{F} \quad 0 \cdot 66$, and, according to Carter, who obtained identical results, identified as the adenosine-2-phosphate and adenosine-3-phosphate. Besides these substances, a smaller spot due to inosinic acid was visible. Adenosine diphosphate obtained by splitting adenosine triphosphate with myosin gave a longer spot, which sometimes seemed to consist of two spots of $R_{F} 0.80$ and $0 \cdot 75$. Adenosine triphosphate from muscle gave only one spot $\left(R_{F}=0.82\right)$, but often this was somewhat extended.

Using our method, we obtained the following components from the adenylic acid preparation, which tentatively have been identified as follows :

\begin{tabular}{lc}
\multicolumn{1}{c}{ Substance } & $R F$ \\
Inosinic acid & 0.86 \\
Adenosine-2-phosphate & 0.69 \\
Adenosine-3-phosphate & 0.61 \\
Adenosine & 0.48
\end{tabular}

The component with $R_{F} 0.69$ has the same $R_{F}$-value as adenosine-5-phosphate, which can be obtained from muscle adenosine triphosphate by cautious hydrolysis or prolonged storage. The components have been identified from the discussions given by Carter $^{1}$ and Kornberg ${ }^{2}$.

Adenosine diphosphate here clearly showed two spots with $R_{F} 0.76$ and 0.69 respectively. The first one is due to the diphosphate; the other one is adenosine-5-phosphate, probably obtained through a small contamination of the myosin by myokinase.

Even adenosine triphosphate from muscle gave two spots, $R_{F} 0 \cdot 83$ and $0 \cdot 76$. The first one is due to the triphosphate, the other to the diphosphate. Adenosine triphosphate is apparently very often contaminated with adenosine diphosphate owing to the instability of the triphosphate.

Our examples show that the modification of the partition coefficient with lauryl amine in amyl alcohol very much facilitates the resolution of the different derivatives of adenosine phosphates, and is superior to Carter's original method.

Orle Snellmax

Bertil Gelotte

'Carter, C. E., J. Amer. Chem. Soc., 72, 1466 (1950).

2 Kornberg, A., and Pricer, jun., W. E., J. Biol. Chem., 186, 557 (195' !.

\section{A Phosphate-absorbing Protein from Muscular Tissue}

IN connexion with the preparation of myosin from cardiac muscle ${ }^{1}$, a protein containing a nucleotide has been isolated. This protein seems to be firmly. bound to the actomyosin complex. However, it is freed to a certain extent when the complex is dissociated into myosin and actin. Only by treatment with organic solvents has it been possible to separate the protein from undissociated actomyosin.

The interesting feature of this substance is its ability to absorb the phosphoric acid residue, which is split from adenosine triphosphate by the enzymatic action of myosin.

The protein is not myosin. It more resembles actin, but it is not an actin in the usual sense. It does not polymerize and does not combine with myosin as actin does.

The present communication records an attempt to analyse the prosthetic group of the protein. This has been done mainly with the aid of paper chromatography and measurements of the ultra-violet absorp. tion spectra in a Beckman spectrophotometer. The absorption curves have a maximum at $260 \mathrm{~m} \mu$ in neutral and alkaline solutions. In acid solutions a maximum at $263 \mathrm{~m} \mu$ is obtained, and here also the curve becomes asymmetric. This is different from that of adenylic compounds. Chromatographic measurements ${ }^{2}$ by the method described elsewhere show that the preparations contain not more than two components, or sometimes only one. In the system 5 per cent dibasic sodium phosphate -0.5 per cent lauryl amine in amyl alcohol, it gives an $R_{F}$ value of 0.67 . In some cases a double spot is obtained with $R_{F} 0.63$ and 0.67 .

After hydrolysis $(0.1 M$ hydrochloric acid, $30 \mathrm{~min}$. at $100^{\circ} \mathrm{C}$.) two components appear in the chromatograms. One of these, with $R_{F} 0 \cdot 40$, is identical with adenine, while the other, with $R_{F} 0 \cdot 85$, has not yet been identified. This component seems to be some pyridine or pyrimidine derivative with basic properties. A prolonged hydrolysis causes no changes in this component, and furthermore the chromatographed substance is soluble in acids only. Milder hydrolysis (alkaline hydrolysis or $0.1 M$ hydrochloric acid, $5 \mathrm{~min}$. at $100^{\circ} \mathrm{C}$.) gives, in addition to the two spots of $R_{F} 0.40$ and $R_{F} 0.85$, two other spots with $R_{F} \quad 0.57$ and $R_{F} \quad 0 \cdot 68$. The former of these may be adenosine-3-phosphate and the latter probably adenosine-5-phosphate. The component with $R_{F} 0 \cdot 68$ 